cell content for analysis. Block retrieval times compared favourably due to the block retrieval fee available, a model which was continued with the Medical Benefits Scheme (MBS) item fee for block retrieval for Australian laboratories, which are also likely to be improved when the tissue test becomes superseded in certain clinical circumstances with the adoption of liquid biopsies.

Conflicts of interest and sources of funding: This study was supported by Roche Products Pty Limited Australia. The authors have received advisor/research funding from Roche, BMS, Bayer, Amgen, MSD, Novartis, and AstraZeneca.

Scott Mead ${ }^{1,2}$, Mark Lucas ${ }^{3}$, Jia-Min Pang ${ }^{4}$, Andrew Fellowes ${ }^{4}$, James Harraway ${ }^{5}$, Suzanne Svobodova ${ }^{6}$, Benhur Amanuel ${ }^{7,8}$, Stephen Fox ${ }^{4,9}$

${ }^{1}$ SydPath Pathology, St Vincent's Hospital, Sydney, NSW, Australia; ${ }^{2}$ Department of Medical Genomics, Royal Prince Alfred Hospital, Sydney, NSW, Australia; ${ }^{3}$ Department of Epidemiology and Preventative Medicine, Monash University, Melbourne, Vic, Australia; ${ }^{4}$ Department of Pathology, Peter MacCallum Cancer Centre, Melbourne, Vic, Australia; ${ }^{5}$ Sullivan Nicolades Pathology, Sonic Healthcare, Brisbane, Qld, Australia; ${ }^{6}$ Molecular Diagnostics, Pathology, Austin Hospital, Studley Road, Heidelberg, Vic, Australia; ${ }^{7}$ School of Pathology and Laboratory Medicine, University of Western Australia, Perth, WA, Australia; ${ }^{8}$ Ludwig Institute of Cancer Research, Melbourne Centre for Clinical Sciences, Austin Health, Melbourne, Vic, Australia; ${ }^{9}$ Peter MacCallum Department of Oncology, University of Melbourne, Melbourne, Vic, Australia

Contact Prof Stephen Fox.

E-mail: stephen.fox@petermac.org

1. Westwood M, Joore M, Whiting P, et al. Epidermal growth factor re ceptor tyrosine kinase (EGFR-TK) mutation testing in adults with locally advanced or metastatic non-small cell lung cancer: a systematic review and cost-effectiveness analysis. Health Technol Assess 2014; 18 $1-166$.

2. Coory M, Fong K, Bowman R, Hall L. Why we need a populationbased approach to clinical indicators for cancer: a case study using microscopic confirmation of lung cancer in Queensland. Intern Med J 2006; 36: 389-92.

3. Hiscock R, Bauld L, Amos A, Fidler JA, Munafò M. Socioeconomic status and smoking: a review. Ann NY Acad Sci 2012; 1248: 107-23.

4. Peters MJ, Bowden JJ, Carpenter P, Lewis J, Solomon B. Outcomes of an Australian testing programme for epidermal growth factor receptor mutations in non-small cell lung cancer. Intern Med J 2014; 44: 575-80.

5. Russell PA, Rogers T-M, Solomon B, et al. Correlation between molecular analysis, diagnosis according to the 2015 WHO classification of unresected lung tumours and TTF1 expression in small biopsies and cytology specimens from 344 non-small cell lung carcinoma patients. Pathology 2017; 49: 604-10.

6. Stone E, Allen HA, Saghaie T, et al. High proportion of rare and compound epidermal growth factor receptor mutations in an Australian population of non-squamous non-small-cell lung cancer. Intern Med J 2014: 44: 1188-92.

7. Kim RH, Lapuk A, Harraway J, et al. Prevalence of the EGFR T790M and other resistance mutations in the Australian population and histopathological correlation in a small subset of cases. Pathology 2020; 52: 410-20.

8. Wong SQ, Li J, Tan AY-C, et al. Sequence artefacts in a prospective series of formalin-fixed tumours tested for mutations in hotspot region by massively parallel sequencing. BMC Med Genomics 2014; 7: 23.

9. The Pharmaceutical Benefits Scheme: Drug Utilisation Sub-Committee (DUSC). Erlotinib and gefitinib: 24 month predicted versus actual analysis. Feb 2017. https://www.pbs.gov.au/industry/listing/ participants/public-release-docs/2017-02/tkis-nsclc-24-month-reviewdusc-prd-2017-02.pdf.

10. Cancer Genome Atlas Research Network. Comprehensive molecular profiling of lung adenocarcinoma. Nature 2014; 511: 543-50.

DOI: https://doi.org/10.1016/j.pathol.2021.01.017

\section{Spindle cell/sclerosing rhabdomyosarcoma with a novel YAP1-MAML2 fusion in a 1-year- old: not all strongly TRK- expressing spindle cell sarcomas in infants are infantile fibrosarcomas!}

Sir,

Strong and diffuse pan-tropomyosin receptor kinase (TRK) reactivity in the setting of paediatric spindle cell tumours can be a diagnostic challenge, considering pan-TRK is not entirely specific for infantile fibrosarcoma or NTRK-rearranged spindle cell neoplasms. Recently, we encountered a challenging case of a spindle cell/sclerosing rhabdomyosarcoma (SRMS) with strong and diffuse pan-TRK reactivity. In addition, we found a new YAP1-MAML2 fusion, to our knowledge not yet described in SRMS.

Ethics approval was granted by the Medical Ethics Committee UGent/UZGent (5/10/2020, EC/088-2020/sds).

Parents of a 1-year-old boy, with a history of a nodule in the musculus latissimus dorsi below the left scapula since birth, noticed recent growth of the mass. Magnetic resonance imaging (MRI) showed a heterogenic lesion $(48 \times 24 \times 38$ $\mathrm{mm}$ ) compatible with an aggressive fibrous mesenchymal lesion. Additional computed tomography (CT) of the thorax and an ultrasound of the abdomen did not show other lesions, or arguments for distant metastases. A Tru-Cut needle biopsy of the lesion was performed. Microscopy showed a cellular proliferation of spindle cells arranged in intersecting long fascicles with focal herringbone growth pattern. Focal haemangiopericytoma-like areas were seen. The cellular spindle cell areas alternated with more fibrous/sclerosing regions (Fig. 1). The spindle cells had elongated, fusiform nuclei with small inconspicuous nucleoli and surrounding indistinct, eosinophilic cytoplasm. Mild cytonuclear atypia, occasional hyperchromatic nuclei and sporadic mitotic activity were noted. Tumour necrosis was absent. In the background, a few scattered lymphocytes were seen. Tadpole or strap cells were not observed. Immunohistochemistry showed diffuse and strong expression of desmin (Fig. 2A) and patchy positivity for smooth muscle actin (SMA). There was cytoplasmic staining for $\beta$-catenin. No immunoreactivity was present for $\mathrm{S} 100$, nuclear transducing-like enhancer of split 1 (TLE-1), CD34, CD31, ERG and factor VIII. Strong and diffuse cytoplasmic staining for pan-TRK (clone EPR17341; Roche Diagnostics, Australia) was observed (Fig. 2B), suggestive for the diagnosis of an infantile fibrosarcoma. However, targeted RNA sequencing did not show NTRK1, NTRK2 or NTRK3 fusions (Oncomine Focus Assay; ThermoFisher Scientific, USA). In addition, staining for MyoD1 showed 

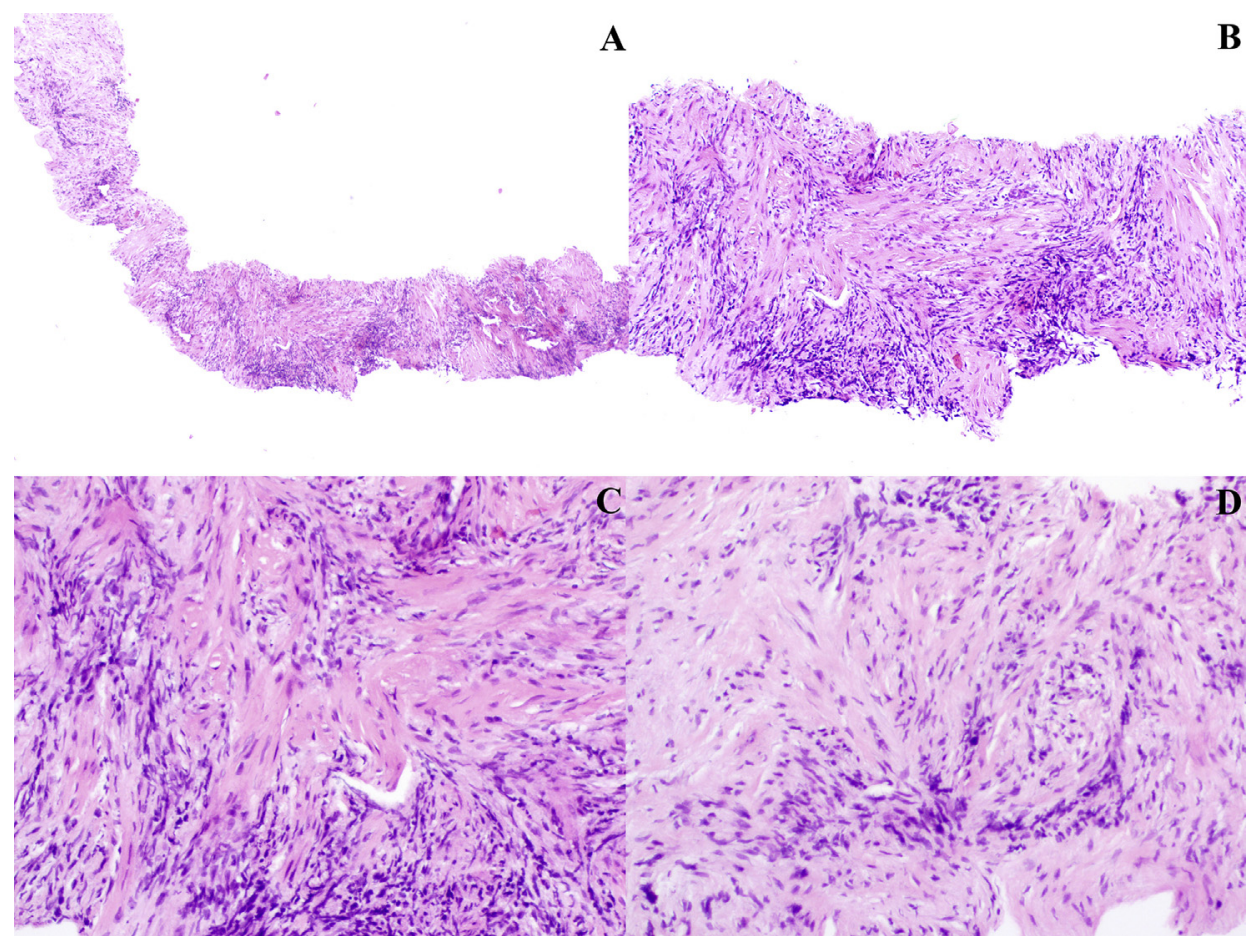

Fig. 1 (A) Tumour with cellular spindle cell areas alternated with more fibrous/sclerosing regions (H\&E). (B) Spindle cells arranged in intersecting long fascicles with focal herringbone growth pattern (H\&E). (C,D) Spindle cells with elongated, fusiform nuclei mild cytonuclear atypia and some hyperchromatic nuclei (H\&E)

diffuse and strong nuclear positivity (Fig. 2C). There was only focal nuclear positivity for myogenin (MYF4) (Fig. 2D).

Based on the spindle cell morphology with variable cytonuclear atypia and intersecting sclerous areas, the clinical presentation (infant with rapidly growing painless mass in head and neck region), as well as the immunohistochemical profile with rhabdomyoblastic differentiation, the diagnosis of SRMS was made. Next, additional bulk RNA sequencing (KAPA hyperprep with ribo-depletion; Roche) was performed, which revealed a YAPl-MAML2 gene fusion. MYOD1 mutations, or rearrangements/fusions of FOXO1, NCOA2 or VGLL2 were not found. After biopsy, chemotherapy was started according to the European Paediatric Soft Tissue Sarcoma Study Group (EpSSG) rhabdomyosarcoma

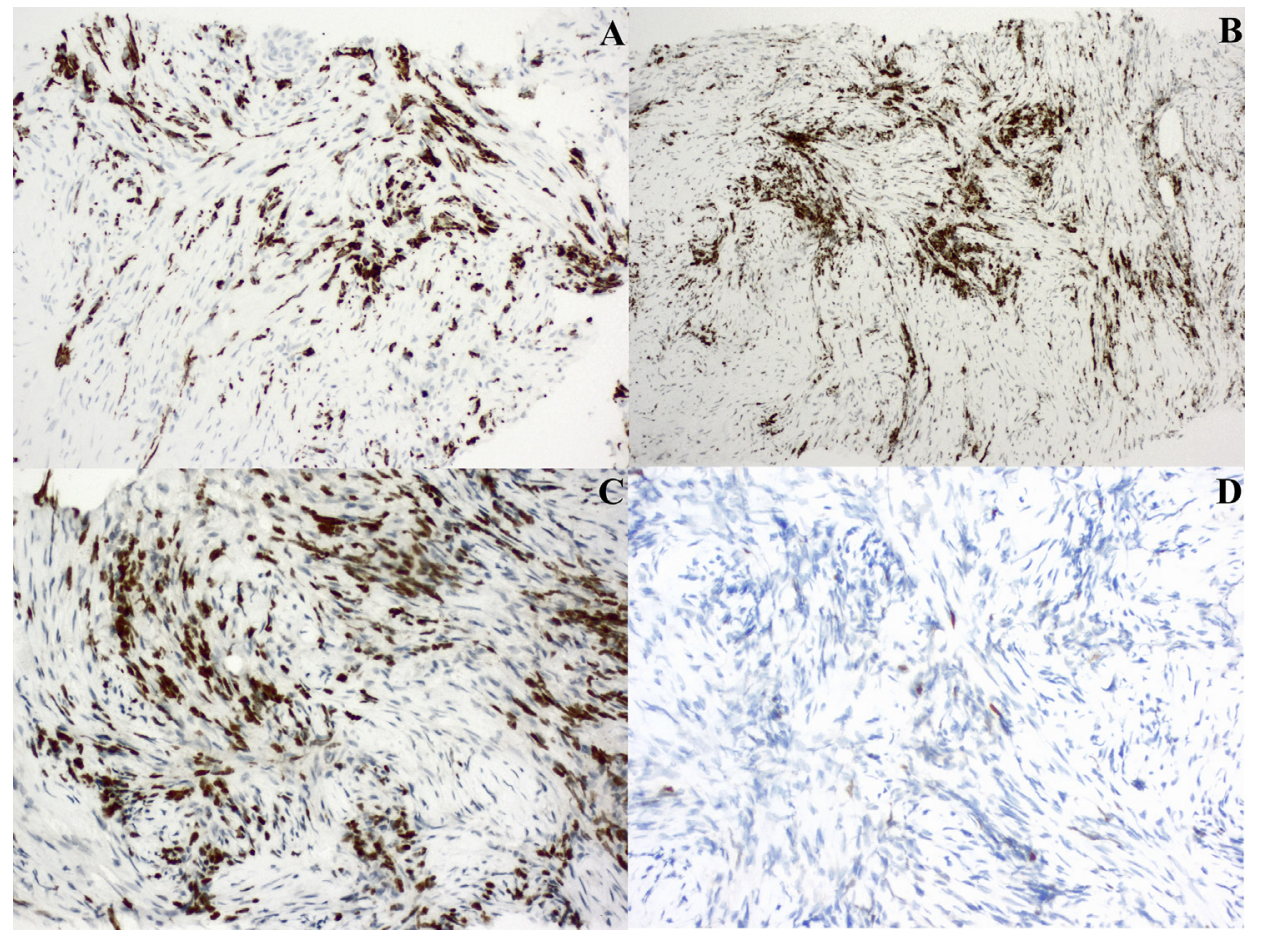

Fig. 2 (A) Diffuse desmin positivity. (B) Pan-TRK showing cytoplasmic expression. (C) Diffuse and strong nuclear positivity for MyoD1. (D) Focal nuclear positivity for myogenin (MYF4). 
(RMS) 2005 protocol [group $\mathrm{C}=$ ifosfamide, vincristine, actinomycin D (IVA) $\times 9$ ]. There was a good clinical response to the neoadjuvant chemotherapy with reduction in tumour size from $48 \mathrm{~mm}$ to $30 \mathrm{~mm}$. After four IVA courses a surgical resection was performed. The post-treatment pathological specimen showed extensive fibrosis, inflammation and atrophic non-neoplastic skeletal muscle. Residual viable tumour cells were seen in $20 \%$ of the resected specimen. The resection margins were tumour free. The post-operative treatment was discussed with clinical experts in childhood RMS. It was decided to give six IVA courses instead of nine, to reduce the long-term toxicity of ifosfamide, followed by three vincristine and actinomycin D (VA) courses. The patient received his last treatment 6 months ago. He is currently in good clinical condition and disease free.

Paediatric spindle cell tumours comprise a heterogeneous group of neoplasms spanning benign to malignant processes. Given the morphological overlap with other paediatric spindle cell tumours, SRMS can be a challenging diagnosis. The differential diagnosis of SRMS can be broad and includes infantile fibrosarcoma, desmoid-type fibromatosis, infantile myofibromatosis, fibrous hamartoma of infancy, lipofibromatosis and lipofibromatosis-like neural tumour. ${ }^{1}$ Due to the atypical spindle cell morphology with fascicular/herringbone pattern, the haemangiopericytoma-like vasculature, scattered lymphocytes in the background, and the strong and diffuse cytoplasmic pan-TRK staining, this case showed a striking resemblance to infantile fibrosarcoma. ${ }^{1}$ There was no co-expression of CD34 and S100, making the diagnosis of a NTRK-rearranged spindle cell neoplasm/lipofibromatosis-like neural tumour less likely. ${ }^{2}$ However, diffuse pan-TRK immunoreactivity, a highly sensitive diagnostic marker for infantile fibrosarcoma and NTRK-rearranged spindle cell neoplasm, is not entirely specific and has been described in a subset of paediatric spindle-cell tumours including infantile myofibromatosis and fibrous hamartoma of infancy. ${ }^{1}$ To the best of our knowledge, pan-TRK immunoreactivity has been described in only one case of SRMS. ${ }^{1}$ Due to the diffuse desmin positivity in this case, MyoD1 and myogenin stainings were performed which led to the diagnosis of an infantile SRMS. Moreover, morphological rhabdomyoblastic differentiation (presence of rhabdomyoblasts with elongated eosinophilic tails with cross striations, so-called tadpole or strap cells) is not detectable in most SRMS (similar to the current case), making the diagnosis of SRMS even more challenging.

SRMS is a subtype of RMS and can be divided into three genomic groups. The first group, the congenital/infantile spindle cell rhabdomyosarcoma, shows gene fusions involving the VGLL2 and NCOA2 genes. The gene fusions include SRF-NCOA2, TEAD1-NCOA2, VGLL2-NCOA2, and VGLL2-CITED2. The second group of SRMS, more common in adolescents and young adults, is characterised by a MYOD1 gene mutation. The third group of SRMS has not shown recurrent identifiable genetic alterations until now., Due to this subclassification with different biological behaviour, additional molecular analysis was performed. ${ }^{4,5}$ Bulk RNA revealed a YAP1-MAML2 fusion [YAP1 (NM_001282101.1) exon 5 - MAML2 (NM_032427.3) exon 2] instead of NTRK, VGLL2 and NCOA2 fusions, and showed no MYOD1 mutations.

In conclusion, we present a challenging case of a rare infantile SRMS with a novel fusion gene and an unexpected strong and diffuse pan-TRK reactivity, strikingly mimicking infantile fibrosarcoma and other paediatric spindle cell tumours. Strong and diffuse pan-TRK reactivity in the setting of paediatric spindle cell tumours is apparently not entirely specific for infantile fibrosarcoma or NTRK-rearranged spindle cell neoplasms; hence, molecular testing/confirmation is strongly advised in these cases. If confronted with paediatric spindle cell lesions with desmin positivity, even in the absence of clear morphological rhabdomyoblastic differentiation, it is recommended to perform additional immunohistochemical stainings for rhabdomyoblastic differentiation (in particular MyoD1 which is more sensitive than myogenin) to confirm/exclude SRMS. Finally, using bulk RNA sequencing, we found a YAP1-MAML2 fusion in this case. Recurrent YAP1-MAML2 fusions have been reported in poroma and porocarcinoma, ${ }^{6}$ and more recently in metaplastic thymoma, ${ }^{7}$ and retiform and composite haemangioendothelioma. ${ }^{8}$ To our knowledge, YAP1-MAML2 fusions have not previously been described in SRMS.

Acknowledgements: We would like to thank Uta Flucke, Patrick Pauwels and Lennart Kester for their valuable technical support.

Conflicts of interest and sources of funding: The authors state that there are no conflicts of interest to disclose.

\section{Fleur Cordier ${ }^{1}$, Eline Ameloot ${ }^{1}$, Catharina Dhooge ${ }^{2}$, Lore Lapeire $^{3,4}$, Gwen Sys ${ }^{4,5}$, Jo Van Dorpe ${ }^{1,4}$, David Creytens ${ }^{1,4}$}

${ }^{1}$ Department of Pathology, Ghent University Hospital, Ghent University, Ghent, Belgium; ${ }^{2}$ Department of Pediatric Hematology-Oncology and Stem Cell Transplantation, Ghent University Hospital, Ghent University, Ghent, Belgium; ${ }^{3}$ Department of Medical Oncology, Ghent University Hospital, Ghent University, Ghent, Belgium; ${ }^{4}$ CRIG, Cancer Research Institute Ghent, Ghent University Hospital, Ghent University, Ghent, Belgium; ${ }^{5}$ Department of Traumatology and Orthopedics, Ghent University Hospital, Ghent University, Ghent, Belgium

Contact Prof Dr David Creytens.

E-mail: david.creytens@uzgent.be

1. Hung YP, Fletcher CDM, Hornick JL. Evaluation of pan-TRK immunohistochemistry in infantile fibrosarcoma, lipofibromatosis-like neural tumour and histological mimics. Histopathology 2018; 73: 634-44.

2. Suurmeijer AJ, Dickson BD, Swanson D, et al. The histologic spectrum of soft tissue spindle cell tumors with NTRK3 gene rearrangements. Genes Chromosomes Cancer 2019; 58: 739-46.

3. Agaram NP, Szuhai K. Spindle cell/sclerosing rhabdomyosarcoma. In: WHO Classification of Tumours Editorial Board. WHO Classification of Soft Tissue and Bone Tumours. 5th ed. Lyon: IARC Press, 2020.

4. Alaggio R, Zhang L, Sung Y, et al. A molecular study of pediatric spindle and sclerosing rhabdomyosarcoma: identification of novel and recurren VGLL2-related fusions in infantile cases. Am J Surg Pathol 2016; 40 224-35.

5. Agaram NP, LaQuaglia MP, Alaggio R, et al. MYOD1-mutant spindle cell and sclerosing rhabdomyosarcoma: an aggressive subtype irrespective of age. A reappraisal for molecular classification and risk stratification. Mod Pathol 2019; 32: 27-36.

6. Sekine S, Kiyone T, Ryo E, et al. Recurrent YAP1-MAML2 and YAP1 NUTM1 fusions in poroma and porocarcinoma. J Clin Invest 2019; 130 3827-32.

7. Vivero M, Davineni P, Nardi V, et al. Metaplastic thymoma: a distinctive thymic neoplasm characterized by YAP1-MAML2 gene fusions. Mod Pathol 2020; 33: 560-5. 
8. Antonescu CR, Dickson BC, Sung YS, et al. Recurrent YAP1 and MAML2 rearrangements in retiform and composite hemangioendothelioma. Am J Surg Pathol 2020; 44: 1677-84.

DOI: https://doi.org/10.1016/j.pathol.2021.02.009

\section{Intra-abdominal Pacinian corpuscle mimicking a peritoneal tumour deposit}

Sir,

Pacinian corpuscles are most commonly encountered in the skin but may be found at other locations, in particular in the peripancreatic mesentery, ${ }^{1}$ where their occurrence may be unexpected and lead to diagnostic confusion. We present a case of peritoneal Pacinian corpuscle identified at laparotomy, which was thought clinically to represent a metastatic tumour deposit.

A 71-year-old woman with no significant past medical history presented with biliary colic and underwent laparoscopic cholecystectomy. Pathologic examination of the gallbladder revealed an unexpected adenocarcinoma [American Joint Committee on Cancer (AJCC) stage pT1b] ${ }^{2}$ arising from intestinal type adenoma. Subsequent staging investigations revealed no evidence of distant metastases and the patient subsequently came forward for segment IVb/V liver resection and portal lymphadenectomy to ensure surgical clearance of the primary. At laparotomy a peritoneal nodule suspicious for metastasis was discovered, and submitted for frozen section examination. Sections from the peritoneal biopsy revealed a Pacinian corpuscle in close association with a nerve (Fig. 1).

Pacinian corpuscles are pressure receptors, hundreds of which are usually located in the dermis of the hand. The corpuscle transmits pressure stimuli via numerous lamellae of connective tissue separated by fluid, to a central partially myelinated nerve fibre. ${ }^{3}$ Anomalous Pacinian corpuscles may include a solitary hypertrophied corpuscle to double or multiple structures, some in varying stages of development. A neuroma can arise from a Pacinian corpuscle; these are usually found in the hand and typically present with pain.

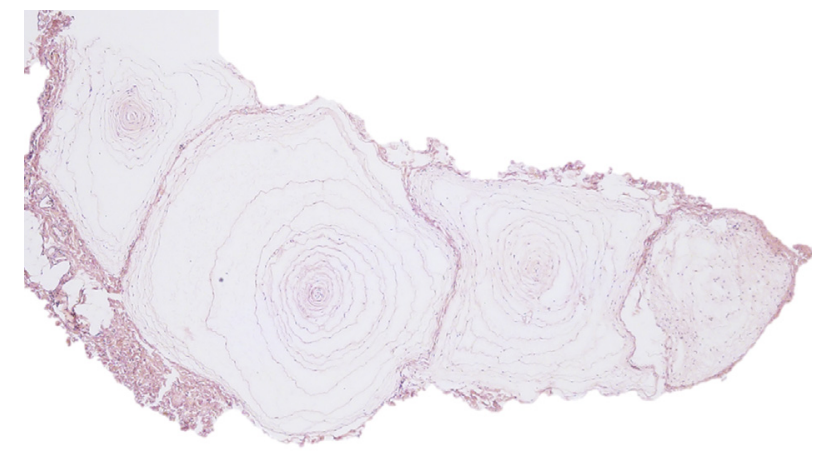

Fig. 1 The tissue submitted for frozen section revealed typical appearances of a Pacinian corpuscle $(\mathrm{H} \& \mathrm{E})$
They have also rarely been reported in the vulva, foot and sacrococcygeal area. ${ }^{3}$ A prior history of trauma or recurrent pressure in the relevant anatomical location has been reported by several authors ${ }^{3,4,6}$ and it is postulated that such trauma to nerves or normal Pacinian corpuscles may be a risk factor for development of a Pacinian corpuscle neuroma. ${ }^{6}$

Dembinski and Jones ${ }^{4}$ describe an intra-abdominal Pacinian neuroma seen macroscopically as white lesions on the adventitia of the aorta, clinically considered suspicious for metastatic colonic adenocarcinoma. This patient had an infrarenal aortic aneurysm and the area may have been subjected to pulsatile pressure/trauma. Stouder and McDonald report a case in which multiple (more than 30) pale nodules were present on the serosa overlying the site of a previous gastrojejunostomy (for duodenal ulcer), and in the adjacent omentum. It is reported that these nodules macroscopically resembled tumour implants on the serosa, but histological examination confirmed enlarged Pacinian corpuscles. More recently, Standop et $a l^{7}{ }^{7}$ reported histologically confirmed Pacinian corpuscles within the pancreas of a 76-year-old female with cancer in the head of the pancreas, and we have similar cases in our files. The purpose of intra- and peripancreatic Pacinian corpuscles is undetermined but may be involved in the regulation of blood flow, detection of changes in the vibration, pressure and/or distension of the peritoneum or pancreas and possibly in the perception of abdominal pain. ${ }^{8}$

In summary, Pacinian corpuscles may be found at unusual anatomical locations and may be confused clinically with tumour deposits; however, thanks to their characteristic appearance, frozen section examination can elucidate the true nature of such lesions, allowing planned surgery to proceed. In particular, pathologists and surgeons should be aware of their frequent occurrence in peripancreatic tissues.

Conflicts of interest and sources of funding: The authors state that there are no conflicts of interest to disclose.

\section{Anna Eccles ${ }^{1}$, Chris Hemmings ${ }^{1,2}$}

${ }^{1}$ Canterbury Health Laboratories, Christchurch, New Zealand; ${ }^{2}$ Christchurch Clinical School, University of Otago School of Medicine, Christchurch, New Zealand

Contact A/Prof Chris Hemmings.

E-mail: chris.hemmings@cdhb.health.nz

1. Stouder DJ, McDonald LW. Enlarged intra-abdominal Pacinian corpuscles simulating tumour implants. Am J Clin Pathol 1968; 49: 79-83.

2. Amin M, editor. AJCC Cancer Staging Manual. 8th ed. New York: Springer, 2017; 303-9.

3. Shah P, Kareliya H, Salome, Toprani T. Pacinian corpuscle tumour. Int J Med Health Res 2019; 5: 48-51.

4. Dembinski AS, Jones JW. Intra-abdominal Pacinian neuroma: a rare lesion in an unusual location. Histopathology 1991; 19: 89-90.

5. Narayanamurthy VB, Winston AT, Gupta A. A rare case of Pacinian corpuscle neuroma. Can J Plast Surg 2005; 13: 43-5.

6. Zanardi F, Cooke RMT, Maiorana A, et al. Is this case of a very rare disease work-related? A review of reported cases of Pacinian neuroma. Scand J Work Environ Health 2011; 37: 253-8.

7. Standop J, Ulrich A, Schneider MB, Andren-Sandberg A, Pour PM. Pacinian corpuscle in the human pancreas. Pancreas 2001; 23: 36-9.

8. Garcia-Suarez O, Calavia MG, Perez-Molto FJ, et al. Immunohistochemical profile of human pancreatic pacinian corpuscles. Pancreas 2010; 39: 403-10. 